\title{
Epigenetics in Bladder Cancer: A Review of the Literature
}

\author{
(1) Tunay DOĞAN, ${ }^{1,2}$ (1) Hülya YAZICl \\ 'Division of Cancer Genetics, Department of Basic Oncology, Istanbul University Institute of Oncology, Istanbul-Turkey \\ ${ }^{2}$ Department of Basic Oncology, Institute of Health Sciences, Istanbul University, Istanbul-Turkey \\ ${ }^{3}$ Department of Medical Biology and Genetics, Istanbul Arel University Faculty of Medicine, Istanbul-Turkey
}

\begin{abstract}
SUMMARY
Bladder cancer $(\mathrm{BC})$ is the $10^{\text {th }}$ most frequent type of cancer worldwide, with around 549.000 new cases and 200.000 deaths/year and it has the highest incidence rates with a strong male and elderly predominance. Besides, BC is a significant burden on health-care systems in terms of long-term therapy due to its high risk of recurrence. The investigation for a biomarker that high sensitivity and specificity to be used in the diagnosis and follow-up of BC is still ongoing because cystoscopic imaging, which is currently using it as the gold-standard technique, is an invasive procedure. Opportunely, the recent epigenetic-based studies are provided successful results for this subject. Therefore, in this study, we review that the DNA methylation and microRNAs relationship with BC were investigated in the light of current studies in the literature, particularly using the meta-analyses. The evaluation of the association of $\mathrm{BC}$ with DNA methylation showed that the hypermethylation was parallel with the muscle invasion, increased tumor stage and grade, poor prognosis, and cancer-associated mortality. Other than, many researchers suggest that various genes or gene panels as the methylation-based biomarkers for use in the follow-up of BC. Similarly, the miRNA panels may successfully differentiate BC samples from healthy controls, and provide successful estimations for survival and recurrence. To all appearances, the increase of the number of epigenetic-based research in $\mathrm{BC}$ not only will provide useful data for clarifying the $\mathrm{BC}$ progression, but will also increase the number of biomarkers with high sensitivity and specificity.
\end{abstract}

Keywords: Biomarker; bladder cancer; DNA methylation; epigenetics; microRNA.

Copyright $\odot$ 2022, Turkish Society for Radiation Oncology

\section{Introduction}

Among all urological malignancies, with approximately 549.000 new cases and 200.000 mortality annually, bladder cancer $(\mathrm{BC})$ is the $10^{\text {th }}$ most common type of cancer worldwide. The prevalence is four-fold higher among males compared with females and is frequently detected in the $6^{\text {th }}$ and $7^{\text {th }}$ decades of life. The incidence and mortality rates in men are 9.6 and 3.2/100.000, respectively, and these data place $\mathrm{BC}$ among the $6^{\text {th }}$ most common cancer in men, and $9^{\text {th }}$ in cancer-related deaths among men.[1] Smoking, alcohol use, chronic exposure to en- vironmental carcinogens, and the parasite that is called Schistosoma haematobium, are shown as the main risk factors in the etiology of BC.[2]

Bladder tumors originate from the urothelium, which is the epithelial layer covering the inner surface of the bladder at a rate of $90 \%$, and at the time of diagnosis, $75 \%$ of the cases have non-muscle-invasive BC (NMIBC) limited to the mucosa. [3,4] On the other hand, $10-20 \%$ of cases with NMIBC generally progress to a poor prognosis, muscle-invasive $\mathrm{BC}$ (MIBC) with a 5 -year survival rate of $60 \%$ for cases with local tumors, and $10 \%$ for metastatic cases.[4] Higher survival rates can be achieved in NMIBCs with transurethral resection
Received: July 06, 2021

Accepted: October 13, 2021

Online: December 08, 2021

Accessible online at:

www.onkder.org

OPEN ACCESS This work is licensed under a Creative Commons

Attribution-NonCommercial 4.0 International License.

\section{Dr. Hülya YAZICI}

İstanbul Arel Üniversitesi Tıp Fakültesi,

Tıbbi Biyoloji ve Genetik Anabilim Dalı,

İstanbul-Turkey

E-mail: hulyayazici@arel.edu.tr 
of the bladder tumor (TUR-BT); however, disease recurrence often presents as a more aggressive tumor in the first 5 years.[5] All this information reveals the necessity of regular follow-up of patients with BC, especially after TUR-BT. Cystoscopic imaging is still using as the gold-standard method for the diagnosis and follow-up of BC.[3] Cystoscopes are optical instruments that allow visualization of the inside of the bladder by entering from the urethra, and they can also perform tissue sampling with biopsy forceps. However, since imaging with a cystoscope is an invasive procedure, the search for a low-cost and reliable biomarker with high sensitivity and specificity that can be used in the diagnosis and follow-up of patients with $\mathrm{BC}$ is still ongoing. However, the recent epigenetic studies are suggested to provide successful results for BC. Therefore, in this study, we review that the DNA methylation and microRNA relationship with BC were investigated in the light of current studies in the literature, particularly using the meta-analyses.

\section{BC and DNA Methylation}

The most known and most studied of epigenetic studies are DNA methylation. DNA methylation describes the formation of 5-methylcytosine with the addition of methyl group (-CH3) to the carbon atom of the $5^{\text {th }}$ position of the cytosine base. The basis of the regulation of gene expression of DNA methylation is due to the frequent methylation of $\mathrm{CpG}$ islands which are intensively detected in the promoter regions of the genes. In almost all cancer types including BC, the genes behaving as tumor suppressors have gene expression suppression due to hypermethylation on their promoters. However, the $\mathrm{CpG}$ islands associated with the promoters of genes whose expressions are always required to be active such as housekeeping genes are in unmethylated condition. [6]

Many studies are evaluating the DNA methylation changes in BC. Bai et al.[7] investigated the APC gene promoter methylation in a sample of $2214 \mathrm{BC}$ patients, and 665 healthy controls in their wide meta-analysis, and reported that methylation in samples with cancer was significantly increased $(\mathrm{p}<0.01)$ compared with the methylation in healthy tissues, and urine samples. They suggested with these data that $A P C$ gene promoter methylation might be associated with the progression of $\mathrm{BC}$ and suggested that should be evaluated as a noninvasive biomarker that could be analyzed from urine samples.[7] The RASSF1A gene promoter methylation was investigated in a sample of $1588 \mathrm{BC}$ patients, and 720 healthy controls and methylation were found to have significantly increased in BC patients $(\mathrm{p}<0.0001)$ in a wide meta-analysis conducted by Zhan et al.[8] Re- searchers confirmed their results with the methylation analysis results obtained from The Cancer Genome Atlas (TCGA) because there was a mismatch between the age, sex, and smoking habits of the samples included in the meta-analyses, and for eliminating the bias due to differences in methods used in all studies.

Therefore, they compared in accordance with the control groups and stages and revealed to show a positive correlation with their meta-analysis results by evaluating selectively the RASSF $1 A$ gene promoter methylation among the global methylation data studies from BC samples on TCGA. Study results showed that the RASSF1A gene promoter methylation in Stage III and Stage IV bladder tumors were statistically significantly increased $(\mathrm{p}<0.0001)$ compared with tumors in Stage I, and Stage II tumors in accordance with the TNM classification in addition to the comparison with the healthy samples. From this point of view, researchers suggested that RASSF1A gene might be used as a clinical biomarker in the diagnosis of $\mathrm{BC}$ because there was a significant association between $\mathrm{BC}$, and RASSF1A gene promoter methylation.[8] Dai et al.[9] stated in their meta-analysis which evaluated the DAPK promoter methylation and $\mathrm{BC}$ association that there was a statistically significant association $(\mathrm{p}<0.00001)$ between the $\mathrm{BC}$ development risk and DAPK promoter methylation in their evaluation with $1247 \mathrm{BC}$ patients, and 405 healthy controls. Researchers suggested at the end of the study that $D A P K$ promoter methylation was not associated with tumor stage $(\mathrm{p}<0.668)$, however, might be associated with higher tumor stage $(\mathrm{p}<0.011)$.

Chen et al.[10] investigated the promoter methylation of the $C D H 13$ gene in their meta-analysis in a sample consisting of $1017 \mathrm{BC}$ patients, and 265 healthy controls. Researchers reported at the end of the study that there was a statistically significant $(\mathrm{p}<0.001)$ association between $\mathrm{CDH} 13$ gene promoter methylation, and $\mathrm{BC}$ development in both tumor tissue and urine sample level. In addition, researchers reported that $\mathrm{CDH} 13$ gene promoter methylation level was higher among Asians compared with the levels among Caucasians $(\mathrm{p}<0.001)$, and methylation status showed no difference in accordance with sex $(\mathrm{p}=0.053)$. They suggested that $\mathrm{CDH} 13$ gene promoter methylation might be used as a prognostic biomarker because a significant association was detected between the increased $\mathrm{CDH} 13$ gene promoter methylation and high-grade bladder tumors $(\mathrm{p}<0.001)$, muscle-invasive bladder tumors $(\mathrm{p}<0.001)$, and multifocal bladder tumors $(\mathrm{p}=0.032)$. In addition to the association of a single gene methylation status with $\mathrm{BC}$, Yu et al.[11] in their wide meta-analysis and systematic review 
evaluated whether DNA methylation had a prognostic significance for BC, and investigated the DNA methylation-based studies conducted between years 2000 and 2018. After their meta-analysis study, they confirmed that the increased DNA methylation status showed a positive correlation with poor prognosis, and DNA methylation studies had prognostic importance for BC.

The data of TCGA are highly important in bioinformatics-based cancer research. Yang et al.[12] analyzed the data involving 540 different methylated genes obtained from TCGA, and the methylation status of seven consisting of MIR6732, SOWAHC, SERPINI1, OR10W1, OR7G3, AIM1, and ZFAND5 had independently prognostic importance for $\mathrm{BC}$ after conducting two variable and single variable Cox regression analyses. Interestingly, the SOWACH gene methylation and the mRNA expression level of the gene showed a negative correlation among these genes; however, SOWACH gene methylation and expression showed a positive correlation with prognosis. In another study, Zhang et al.[13] evaluated the microarray data expressing the genes which were included in the Gene Expression Omnibus database, and which showed abnormal methylation and expression in $\mathrm{BC}$ using various bioinformatics tools. The obtained candidate genes after the analysis were compared with the data obtained from TCGA and were confirmed. At the end of the study, the genes CDH1, DDOST, CASP8, DHX15, and PTPRF were mainly overexpressed, and were hypomethylated; however, all the genes as GNG4, ADCY9, NPY, ADRA2B, and $P E N K$ were hypermethylated, and their expressions were mainly decreased. Researchers suggested that these ten genes might be the biomarkers in the evaluation of the diagnosis, treatment, and prognosis of BC.

Bosschieter et al.[14] analyzed the methylation status of protein-coding genes of CADM1, FAM19A4, GHSR, MAL, PHACTR3, PRDM14, SST, and ZIC1, and miRNA genes of miR-124-2, miR-129, miR-137, miR148 , miR-181, and miR-935 in the urine samples of $72 \mathrm{BC}$ patients, and 75 healthy controls using the quantitative MS-PCR. No significant difference was detected except the methylation condition of the CADM1, miR-124-2, miR-137, and miR-181 genes; however, the methylation status of the remaining ten genes was reported to show an increase and a significant difference in BC patients compared with the control group. Researchers found that GHSR and MAL genes could successfully differentiate specifically the urine samples of $\mathrm{BC}$, and healthy individuals with $92 \%$ sensitivity and $85 \%$ specificity in their analysis by creating a panel with the genes statistically significantly strongest among these ten genes.
Then, researchers analyzed the tissue-based methylation status of GHSR, and MAL genes, and showed a significant increase in tumor tissues, and suggested that the methylation analysis of these two genes might be used as a panel that will enable noninvasive diagnosis from urine for BC. Stubendorff et al.[15] evaluated the association of metastasis and lymph node involvement with DNA methylation in MIBC patients in their study. They analyzed the global DNA methylation status in 23 muscle-invasive bladder tumor tissues in the scope of the study in a microarray-based analysis. In addition, they supported their studies by analyzing the protein and mRNA expression levels in wider patient samples, and by performing functional analyses in T24 and 253JB-V cell lines. They reported that the methylation analysis of the panel consisting of 3 genes as KISS $1 R$, $S E P T$, and CSAD might be a strong prognostic indicator that could differentiate the positivity or negativity of lymph node involvement in MIBC patients. Leão et al.[16] evaluated the TERT promoter mutation prevalence, and TERT Hypermethylated oncologic region (THOR) methylation in their study conducted with 237 bladder tumor tissue, and 94 healthy bladder tissues. They stated that THOR hypermethylation is a risk factor for NMIBC due to its increase of TERT expression, and the altogether analysis of TERT promoter mutation and THOR hypermethylation could provide statistically significant information about recurrence $(\mathrm{p}<0.0001)$, and progression $(\mathrm{p}=0.025)$.

Guo et al.[17] emphasized that one of the most significant symptoms of $\mathrm{BC}$ of hematuria was among the primary causes of presenting to the clinic, however, was mostly idiopathic, and with a different perspective aimed to identify malignity in the case of hematuria by investigating the promoter methylation of ten candidate genes involving the $A B C C 6, B R C A 1, C D H 1$, GDF15, HSPA2, RASSF1A, SALL3, THBS1, TMEFF2, and VIM genes from the urine samples of 217 patients with urothelial carcinoma in the upper urinary system, and urothelial BC with hematuria, and from the samples of 256 healthy controls using the MS-PCR. The analysis of the promoter methylation of eight genes of VIM, CDH1, SALL3, TMEFF2, RASSF1A, BRCA1, GDF15, and $A B C C 6$ out of ten candidate genes could differentiate the presence of urothelial carcinoma in hematuric patients with $83 \%$ sensitivity, and $60 \%$ specificity and the panel consisting of the genes CDH1, HSPA2, RASSF1A, TMEFF2, VIM, and GDF15 could differentiate upper urinary tract urothelial carcinoma from other groups with $82 \%$ sensitivity and $68 \%$, and the panel consisting of the genes VIM, RASSF1A, GDF15, and TMEFF2 could 
differentiate urothelial BC from other groups with $82 \%$ sensitivity, and $53 \%$ specificity. In addition, researchers indicated that the panel consisting of $C D H 1, S A L L 3$, THBS1, TMEFF2, VIM, and GDF15 genes showed a higher success for detecting malignity in patients with hematuria in the accompany with urine cytologic analysis. van Kessel et al.[18] prospectively included 1239 NMIBC patients ( 276 are low risk, 273 are moderate risk, and 555 are high risk of tumor progression) into their multicenter study with the participation of researchers from 6 European countries by emphasizing that the European Urology Association recommended the performing of pathologic parameters-based risk classification in the guidelines for NMIBC which constitute more than $70 \%$ of bladder tumors. Researchers investigated the methylation status of GATA2, TBX2, TBX3, and ZIC4 genes, and the mutation status of FGFR3, TERT, $P I K 3 C A$, and RAS genes in the fresh frozen tumor tissues of patients. 54 patients were found to show progression toward MIBC, among all genes the mutation status of FGFR3 gene and methylation of GATA2 and TBX3 genes were shown to be significantly associated with the disease progression, and researchers indicated that these genes might be a risk factor that could be used in the estimation of progression in NMIBC patients. Shindo et al.[19] in their two centers prospective study, included 132 NMIBC patients initially, and taken urine samples, and followed up these patients, and analyzed the methylation status of miR-9-3, miR-124-2, miR-124-3, and miR-137 genes in all samples compatible with the study criteria by taking repeat urine sample in recurrent cases using the bisulfite pyrosequencing. Researchers investigated the association between the methylation status in these miRNA genes, and recurrence, and revealed that urine-based methylation analysis conducted using this miRNA gene panel might be an indicator for the decision of recurrence and radical cystectomy in BC. López et al.[20] performed genome-wide methylation analysis in different stages and grades 70 bladder tumors, and ten healthy tissue samples and the tumor tissues of 43 genes reported to have hypermethylation higher than $10 \%$. Researchers found that the JAK3 gene hypermethylation and nonmethylation of EYA4, GAT6, and SOZ1 genes were associated with low-grade non-invasive bladder tumors. However, the hypermethylation of the genes CSPG2, HOXA11, HOXA9, HS3ST2, SOX1, and TWIST1 was found associated with muscle invasion. Researchers reported that the hypermethylation of the genes APC, CSPG2, EPHA5, EYA4, HOX9, IPF1, ISL1, JAK3, PITX2, SOX1, and JAK3 might be used in the estimation of cancer-associated survival, and the hyperme- thylation of SOX1, PITX2, or CSPG2 genes was significantly associated with $\mathrm{BC}$ associated mortality risk.

The evaluation of the response to Bacillus CalmetteGuérin (BCG) immunotherapy which is an important treatment modality in NMIBC patients is highly important in the decision of further treatment options in later stages. Husek et al.[21] investigated the DNA methylation status of tumor suppressor genes in response to BCG immunotherapy in their study. Researchers included 82 NMIBC patients with high grades in their study and divided patients into two groups as successful or unsuccessful in accordance with the response to BCG immunotherapy. Analyses associated with methylation changes were performed using the commercial ME001 (MRC-Holland Inc.) and ME004 (MRC-Holland Inc.) MS-MLPA kit which includes probe set for various tumors suppressor genes using the paraffin-embedded tumor tissues of patient groups, and 13 healthy bladder tissues. Researchers showed in the study that the methylation status of tumor suppressor $C D K N 2 b$, MUS81a, PFM-1, MSH6, and THBS1 genes was significantly associated with immunotherapy response, and methylation of CDN2b and MUS81 $a$ genes was statistically significantly associated with $(\mathrm{p}=0.0312, \mathrm{p}=0.0191)$ successful response to BCG immunotherapy, however, showed that $C D N 2 b$ and MUS81 $a$ genes were not methylated in the patient group who responded unsuccessfully to BCG immunotherapy.

Although smoking is a primary risk factor in BC, patients were divided into subgroups and evaluated in accordance with their smoking habits in a very limited part of studies in the literature. The interaction of epigenetic factors with the environment is a well-known fact, however particularly in DNA methylation, it is clear that smoking will create a significant variation for $\mathrm{BC}$ development, and progression between individuals. The first clinical and in vitro study evaluating this issue was performed by Jin et al.[22] Researchers analyzed the significant changes in the tissue samples and urine sample metabolomes of 78 smokers and 41 nonsmoker BC patients using the LC-MS, and evaluated the obtained data with bioinformatics tools, and confirmed with the cell lines and TCGA data. Significantly higher methylated metabolites, polycyclic aromatic carbons, deteriorated nucleotides/adducts in DNA, and genetic changes showing the DNA damage were detected in the smoker BC patient group compared with the nonsmoker group. These data explain the detection of the higher number of advanced-stage tumors in the presence of smoking at the molecular level. Researchers also reported that DNMT1 enzyme (and therefore smoking- 
induced DNA methylation) was increased in smoker BC patients, and the aniline level which is important in bladder carcinogenesis, and SAM level directly associated with DNA methylation were found significantly higher in the smoker group; however, the S-AdenocylL-Homosistein level was significantly decreased.[22]

Epidemiologic studies showed that the prevalence of BC was lower among Afro-Americans compared with other people in the same region, and this difference was interpreted as that $\mathrm{BC}$ prevalence might be lower in the black race. Vantaku et al.[23] suggested that this was stemmed from DNA methylation-associated epigenetic changes, and they analyzed the DNA methylation patterns of Afro-American BC patients, and Euro-American BC patients using bisulfite sequencing, and using LC-MS/MS for metabolomes, and compared the values. In addition to the metabolomic differences, the study results showed that the CpG islands of the promoters of xenobiotic enzymes known as important in $\mathrm{BC}$ progression were more commonly hypermethylated in Afro-Americans compared with the Euro-Americans. Researchers revealed at the end of the study that the race and ethnicity differences in the molecular biology of $\mathrm{BC}$ might create prognostic variations over DNA methylation.

\section{BC and MicroRNAs}

MicroRNAs are short and noncoding RNA molecules with a length of 18-28 bp whose target molecules are mRNAs. MicroRNAs represent a very small portion of the total RNA despite taking more than half of all genes in their targets. In addition, the interaction ability of a single microRNA with multiple mRNAs increases the functionality of these molecules. MicroRNAs are exposed to a complex maturation period in the nucleus, and molecules in the form of pre-miRNA are transferred to ultimate miRNA form in the cytoplasm through molecular exportin mediated transfer. MicroRNAs are commonly suggested to suppress the transcription by binding to 3'-UTR regions of mRNAs; however, conducted studies showed that they might increase the transcription.[24,25] Considering all these features, the suppression of oncogene-associated miRNAs with anti-miRNAs (antagomir) or strategies such as stimulation of the tumor-suppressing genes as an option in cancer treatment has come to agenda for increasing the chemoradiotherapy sensitivity.[25]

One of the most comprehensive studies on the importance of miRNAs in BC is the meta-analysis conducted by Xie et al.[26] The researchers collected and filtered all studies conducted between 2009 and 2016 from different databases, and investigated 37 miRNAs which were reported to have prognostic importance in the sample consisting of 2753 BC patients, and in different stages. The meta-analysis showed that 6 miRNAs consisting of miR-21, miR-143, miR-153, miR-200, miR-214, and miR-222 were found to have been commonly repeated in most studies. They reported that miR-21 and miR-222 expressions showed correlation with shortened general survival, and increased miR143 and miR-155 expression were associated with shortened nonprogressive survival and decreased miR-214 expression was associated with shortened recurrence-free survival and stated that this miRNA panel might be evaluated as the useful biomarkers and therapeutic targets in the follow up of BC patients.[26] Chen et al.[27] investigated in another meta-analysis the association of miRNA levels in kidney, bladder, and prostate urologic cancers with survival estimation. Researchers investigated 80 different miRNAs in 151 data set after collecting and filtering all miRNAbased studies conducted for three urologic cancer types in different databases until the end of 2017 and confirmed their results with the TCGA data. The changes in miR-21, miR-34a, miR-141, and miR-203 were commonly observed for each 3 urologic cancer types however after TCGA confirmation only miR21 showed statistically higher significantly $(p<0.001)$ shortened general survival. In addition, miR-21 was found associated with poor prognosis in BC independent of other cancers. Researchers indicated that the evaluation of miRNA based indicators as panels would have higher success rates compared with their single evaluation, and showed that the panel in kidney cancer consisting of miR-27a, miR-27b, miR-141, miR-144, miR-497, and miR-942 could estimate a statistically significant general survival $(\mathrm{p}<0.001)$ compared with each miRNA in the panel. Yang et al.[28] investigated how the single miR-15a affected the general survival in 18 different cancer types in their meta-analysis, and gathered all studies performed until the end of 2018, and included 1616 cancer patients in ten articles to their study after filtering. The decreased miR-15a expression was found associated with statistically significantly shortened general survival (for BC, $\mathrm{p}=0.0081$ ) in 8 cancer types including $\mathrm{BC}$ in the meta-analysis, and researchers suggested that miR-15a might be used as a biomarker in the estimation of general survival.

Although miRNA panels in different combinations may have different sensitivity, and specificity rates for survival estimation, in a general sense the question of whether miRNAs could be used for BC diagnosis is an important question. In their meta-analysis, Shi et al.[29] included ten articles in their studies after filtering and investigated the diagnostic importance of 
miRNAs in a sample consisting of $1556 \mathrm{BC}$ patients and 1347 healthy controls. Researchers reported after the meta-analysis that miRNA profiling from blood could show high success in $\mathrm{BC}$ diagnosis however, ethnic differences might cause significant variations even in the same sample subgroups. Researchers also revealed that miRNA profiling particularly from blood in Asian population-based studies showed higher accuracy for $\mathrm{BC}$ diagnosis however, more studies were required for exact results. Xiao et al.[30] in their more detailed meta-analysis included 22 articles after filtering and investigated whether the quantitative RT-PCR based miRNA profiling performed from blood, and urine in the samples of 4558 BC patients, and 4556 healthy controls might be used as a noninvasive biomarker for $\mathrm{BC}$ diagnosis. Researchers concluded that the blood and urine-based miRNA analysis could differentiate BC patients from healthy controls with $74 \%$ sensitivity and $78 \%$ specificity, and could detect the early period $\mathrm{BC}$, blood-based analyses alone showed higher performance compared with the urine-based analyses, the use of miRNAs as panels increased the performance, and miRNA profiling in Caucasians showed lower success in $\mathrm{BC}$ diagnosis compared with the Asian populations.

In their meta-analysis, Ouyang et al.[31] investigated the diagnostic value of miRNA profiling in kidney, bladder, and prostate urologic cancers, and included 39 articles in their study after filtering. The general sensitivity and specificity of miRNA profiling in the diagnosis were found as $74 \%$, and $72 \%$, respectively for three urologic cancer types. In the independent analysis conducted for $\mathrm{BC}$, miRNA profiling in a general sense could differentiate $\mathrm{BC}$ samples from healthy controls with $74 \%$ sensitivity and $74 \%$ specificity; the sensitivity reached $79 \%$, and specificity to $73 \%$ in Asians in the analysis considering the ethnical differences, and sensitivity levels were detected as $71 \%$, and specificity as $71 \%$ in Caucasians. The diagnostic value of miRNA profiling was also investigated in the blood, and nonblood samples in BC, and $77 \%$ sensitivity, and $74 \%$ specificity were found in blood-based miRNA profiling; however, $74 \%$ sensitivity and $72 \%$ specificity level was obtained in nonblood samples. Researchers also revealed that $73 \%$ sensitivity and $71 \%$ specificity level were detected in the use of single miRNA in BC diagnosis, and the use of miRNA panel increased the level of diagnostic sensitivity and specificity to $83 \%$.[31] There is a consensus on the preference of miRNA panels for differentiative diagnosis of $\mathrm{BC}$ for survival estimation for increasing the performance; however, it was emphasized that the investigation of the effect of the use of single miRNAs for $\mathrm{BC}$ progression, treatment response, or tumor behavior is another topic that should be evaluated.
Conducted studies showed that the expressional changes of miRNAs also had huge roles on the key genes included in the two pathway hypothesis in addition to the role of mutations. To exemplify, the miRNA-99a and miR-100 are known to have roles in the regulation of the FGFR3 gene products, the increased miR-143 resulted in the decrease in the RAS gene expression; miR-125b, miR-143, miR-30a/c, and miR-223 directly targeted the TP53 gene, and miR-10, and miR-129 caused an indirect change in the regulation of TP53 gene by targeting the MDM2, MDM4, and ATM genes.[32] In this regard, the miRNA profiling in the early stage $\mathrm{BC}$ might change the treatment modalities by rapidly distinguishing the MIBC or NMIBC differentiation, and might emerge strong therapeutic targets. The gene product enzyme of METTL3 is suggested to have a role in all stages starting from their being transcribed on RNAs and affected the tumor development by regulating the N6-Methyladenosine (m6A) modification on the mRNAs of critical oncogenes, and tumor suppressors, and specifically caused the $\mathrm{BC}$ progression through $\mathrm{m} 6 \mathrm{~A}$ dependent AFF4/NF- $\mathrm{kB} / \mathrm{MYC}$ signal pathway.[33,34] Based on this data, Han et al.[34] investigated how the METTL3 affected the m6A modification in noncoding RNAs such as miRNA in the concept of BC and used the EJ and T24 MK cell lines in addition to tumor tissues and healthy tissues adjacent to tumor in BC patients in their study. Study results showed that METTL3 had an expressional increase correlated with poor prognosis in BC. Researchers also suggested that METTL3 accelerated the pri-miR-221 and pri-miR-222 maturation through an m6A dependent pathway, and this was useful in decreasing PTEN expression which is significantly responsible for the progression of $\mathrm{BC}$ cells and suggested its use in $\mathrm{BC}$ treatment. In another study, Lu et al.[35] evaluated the effect of a noncoding circular RNA of circSLC8A1 in $\mathrm{BC}$ progression and used the cell lines consisting of 5637, T24, J82, EJ, UMUC, RT4, and SV-HUC-1 in addition to tumor tissue and healthy tissues in the adjacent to tumor in BC patients. Researchers revealed that circSLC8A1 expression significantly decreased in cancerous cell lines, and particularly in tumor tissues, and this decrease showed a positive correlation with tumor stage and grade. In addition, they showed that circSLC8A1 suppressed the $\mathrm{BC}$ progression by inhibiting the expression increase of PTEN expression of these miRNAs by directly interacting with miR-130b, and miR-494. Hou et al.[36] evaluated the effect of miR-127 on BC progression in their study. First, the researchers showed with the bioinformatics analysis that miR-127 targeted the $K M T 2 D$, and investigated this data using the NMIBC tissue of 20 patients, and MIBC tissues of 20 patients, and the cell lines consisting of RT4, T24, UMUC-3, 
5637, EJ, and SV-HUC-1. Study results showed that the miR-127 expression was found higher in MIBC tissues compared with the expression in NMIBC tissues, and in addition, an increase in $\mathrm{BC}$ cell lines was detected compared with the level in healthy cell lines. The silencing of miR-127 expression significantly decreased the viability and migration in T24, and UMUC-3 cells. In addition, researchers stated that $K M T 2 D$ was associated with a good prognosis by demonstrating that KMT2D which has a role in all genome transcription of miR-217 primarily the H3K4me1, increased its expression.

Lin et al.[37] used the tissue samples, and T24, RT4, 5637, HT-1376, J82, UMUC-3, and TCCSUP cell lines of $164 \mathrm{BC}$ patients, and investigated whether miR-325 might be a potential biomarker for BC. Study results showed that the expression of the miR-325 in tumor tissues, and cancerous cell lines were statistically significantly decreased compared with the level in healthy tissues and cell lines ( $\mathrm{p}<0.05)$, and miR-325 expression was found associated with statistically significantly decreased $(\mathrm{p}=0.0114)$ postoperative general survival. In addition, the researchers showed that miR-325 was found to show tumor suppressor features with the decrease of cisplatin chemoresistance with tumor proliferation and migration in vitro experiments by overexpression through lentiviral transfection in T24, and 5637 cell lines; however, in vivo experiments the transplant growth and GO/G1 transition in cells were statistically significantly delayed in the group where the miR-325 was overexpressed in T24 and 5637 cells subcutaneously implanted to athymic-nude nu/nu mice appropriate for transplantation. Tian et al.[38] used the T24, J82, and SV-HUC-1 cell lines in addition to tumor tissues and healthy bladder tissue in the adjacent to tumor obtained from $50 \mathrm{BC}$ patients in their study which investigated the effect of miR-621 in progression in BC. Researchers showed that miR-621 expression level in tumor tissues with T24 and J82 cell lines was statistically significantly highly decreased compared with the healthy tissues and noncancerous SV-HUC-1 cell line $(\mathrm{p}<0.01)$, and this decrease was correlated with the tumor progression. In addition, the miR-621 expression level was shown to have a negative correlation with general survival. Researchers indicated that miR-621 directly targeted the 3'-UTR region of TRIM29. In addition, TRIM29 level was statistically significantly increased in tumor tissues compared with the levels in healthy tissues, and TRIM29 and miR-621 showed a completely reverse directional expressional change is statistically higher significance level in tumor tissues $(\mathrm{p}<0.001)$. In addition, miR-621 was suggested to inhibit the proliferation and metastasis through the $\mathrm{Wnt} / \beta$-catenin signal pathway in BC cells by targeting the TRIM29.
Feng et al.[39] evaluated the miRNA-99a change and its prognostic importance in $\mathrm{BC}$ in the tumor tissues, and healthy bladders tissues adjacent to tumor obtained from $100 \mathrm{BC}$ patients; in the plasma samples obtained from $50 \mathrm{BC}$ patients, and 50 healthy controls, and in J82, HT1376, RT4, T24, TCCSUP, HCV29, and HU609 cell lines. Study results showed that the expression level of miR-99a was found statistically significantly lower in tumor tissues, in cancerous cell lines, and the plasma samples of BC patients $(\mathrm{p}<0.01)$. The decreased miR-99a level was found correlated with the aggressive BC phenotype, and researchers showed that proliferation was correlated with aggressive $\mathrm{BC}$ phenotype, and proliferation in cell lines which overexpressed the miR-99a was inhibited. Wu et al.[40] evaluated the change of miR-379-5p, and their prognostic importance in $\mathrm{BC}$ in tumor tissue, and healthy bladder tissues obtained from $27 \mathrm{BC}$ patients, and in T24, EJ, TCCSUP, and SV-HUC-1 cell lines in their study. Bioinformatics analyses showed that miR-379-5p directly targeted the 3'-UTR region of MDM2; therefore, MDM2 was also included in the study. Study results showed that miR-379-5p expression levels statistically significantly $(\mathrm{p}<0.05)$ decreased in tumor tissues, and T24, J82, and TCCSUP BC cell lines compared with the expression in healthy tissues, and noncancerous SV-HUC-1 cell line; however, the expression level of $M D M 2$ was increased in cancer tissues. Researchers found that cell proliferation was inhibited, and invasion and metastasis capacity was statistically significantly decreased in T24, and EJ cancerous cell lines where the miR-379-5p was overexpressed through transfection $(\mathrm{p}<0.05)$, and they found similar results when they suppressed the MDM2 expression in the same cell lines through siRNA. Jiang et al.[41] investigated the effect of miR-93, and PEDF which targets from its 3 '-UTR region on the $\mathrm{BC}$ proliferation, and invasion in the tumor tissues of $30 \mathrm{BC}$ patients, and healthy bladder tissue in the adjacent to tumor in TCCSUP, 5637, UMUC-3, and T24 cell lines. MiR-93 expression was found to have statistically significantly increased in tumor tissues compared with the expression in healthy tissues $(\mathrm{p}<0.05)$ and showed a correlation with tumor stage, and with the presence of lymph node metastasis. Researchers suppressed the expression of miR-93 in TCCSUP and UMUC- 3 cell lines where the miR-93 expression was the highest among all cell lines and showed that cell proliferation and invasion were statistically significantly $(\mathrm{p}<0.05)$ decreased. In addition, they also revealed that miR-93 expression was decreased in cell lines where the PEDF was overexpressed, and cell proliferation and invasion were statistically significantly decreased $(\mathrm{p}<0.05)$. 


\section{Conclusion}

The evaluation of the association of BC with DNA methylation in the literature showed that hypermethylation was parallel with the muscle invasion, increased tumor stage and grade, poor prognosis, and cancer-associated mortality. Many researchers suggest many different genes or gene panels as the methylation-based biomarkers for use in the follow-up of BC. The clinical use of biomarkers with meta-analyses confirmed high sensitivity and specificity among all these candidate genes provides convenience both for the patient and the physician in the diagnosis, and treatment of BC. In this sense, the EpiCheck ${ }^{\otimes}(\mathrm{Nu}-$ cleix Inc.), which has recently been used in clinics for monitorization of $\mathrm{BC}$ patients is an epigenetic diagnostic test involving the analysis of the methylation change of a panel consisting of 15 genes, and has a $92 \%$ sensitivity and $88 \%$ specificity and has been regarded as a candidate for use as an alternative to urine cytology. It is expected to obtain more accurate data on the sensitivity and specificity with the common use of the test.[42] Literature studies also show that miRNAs have significant importance for BC. The miRNA panels in different combinations obtained after urine, and tissue miRNA profiling may successfully differentiate BC samples from healthy controls, and these panels may provide successful estimations for survival, and recurrence. miRNAs associated with genes that have key roles in $\mathrm{BC}$ progression are highly effective in the development of $\mathrm{BC}$ subtypes. There is various evidence that the proliferation, invasion, and metastatic features of BC cells were suppressed in cell culture-based experiments where the strategic miRNAs were selectively inhibited or overexpressed, and it is clear that the associated miRNAs could be evaluated as therapeutic targets. In addition to all these data, miRNAs are found to have complex associations with other epigenetic mechanisms having a role in $\mathrm{BC}$ considering their relations with other noncoding RNAs and taking the regulators in their targets which are responsible for the histone modification and DNA methylation, and with their interactions with the enzymes responsible from transcription. To all appearances, the increase of the number of epigenetic-based research in $\mathrm{BC}$ not only will provide useful data for clarifying the $\mathrm{BC}$ progression, but will also increase the number of biomarkers with high sensitivity and specificity.
Peer-review: Externally peer-reviewed.

Conflict of Interest: The authors declare no conflicts of interest.

Financial Support: The authors received no financial support for the publication of this article.

\section{References}

1. Bray F, Ferlay J, Soerjomataram I, Siegel R, Torre L, Jemal A. Global cancer statistics 2018: GLOBOCAN estimates of incidence and mortality worldwide for 36 cancers in 185 countries. CA Cancer J Clin 2018;68(6):394-424.

2. Johansson S, Cohen S. Epidemiology and etiology of bladder cancer. Semin Surg Oncol 1997;13(5):291-8.

3. Sanli O, Dobruch J, Knowles M, Burger M, Alemozaffar M, Nielsen ME, et al. Bladder cancer. Nat Rev Dis Primers 2017;3(1):1-19.

4. Kamoun A, de Reyniès A, Allory Y, Sjödahl G, Robertson AG, Seiler R, et al. A consensus molecular classification of muscle-invasive bladder cancer. Eur Urol 2020;77(4):420-33.

5. Bladder cancer: Diagnosis and management of bladder cancer. BJU Int 2017;120(6):755-65.

6. Michalak E, Burr M, Bannister A, Dawson M. The roles of DNA, RNA and histone methylation in aging and cancer. Nat Rev Mol Cell Biol 2019;20(10):573-89.

7. Bai Z, Liu Q, Wang X, Liu W. APC promoter methylation is correlated with development and progression of bladder cancer, but not linked to overall survival: A meta-analysis. Neoplasma 2019;66(3):470-80.

8. Zhan L, Zhang B, Tan Y, Yang C, Huang C, Wu Q, et al. Quantitative assessment of the relationship between RASSF1A gene promoter methylation and bladder cancer (PRISMA). Medicine (Baltimore) 2017;96(7):e6097.

9. Dai L, Ma C, Zhang Z, Zeng S, Liu A, Tang S, et al. DAPK promoter methylation and bladder cancer risk: A systematic review and meta-analysis. PLoS One 2016;11(12):e0167228.

10. Chen F, Huang T, Ren Y. Clinical significance of CDH13 promoter methylation as a biomarker for bladder cancer: A meta-analysis. BMC Urol 2016;16(1):52.

11. Yu Y, Cao H, Zhang M, Shi F, Wang R, Liu X. Prognostic value of DNA methylation for bladder cancer. Clin Chim Acta 2018;484:207-12.

12. Yang Z, Liu A, Xiong Q, Xue Y, Liu F, Zeng S, et al. Prognostic value of differentially methylated gene profiles in bladder cancer. J Cell Physiol 2019;234(10):18763-72.

13. Zhang Y, Fang L, Zang Y, Xu Z. Identification of core genes and key pathways via integrated analysis of gene 
expression and DNA methylation profiles in bladder cancer. Med Sci Monitor 2018;24:3024-33.

14. Bosschieter J, Nieuwenhuijzen J, Hentschel A, van Splunter AP, Segerink LI, Vis AN, et al. A two-gene methylation signature for the diagnosis of bladder cancer in urine. Epigenomics 2019;11(3):337-47.

15. Stubendorff B, Wilhelm K, Posselt K, Catto J, Hartmann A, Bertz B, et al. A three-gene methylation marker panel for the nodal metastatic risk assessment of muscle-invasive bladder cancer. J Cancer Res Clin Oncol 2019;145(4):811-20.

16. Leão R, Lee D, Figueiredo A, Hermanns T, Wild $P$, Komosa $\mathrm{M}$, et al. Combined genetic and epigenetic alterations of the TERT promoter affect clinical and biological behavior of bladder cancer. Int J Cancer. 2018;144(7):1676-84.

17. Guo R, Xiong G, Yang K, Zhang L, He SM, Gong YQ, et al. Detection of urothelial carcinoma, upper tract urothelial carcinoma, bladder carcinoma, and urothelial carcinoma with gross hematuria using selected urine-DNA methylation biomarkers: A prospective, single-center study. Urol Oncol Sem Original Investig 2018;36(7):342.e15-23.

18. van Kessel K, van der Keur K, Dyrskjøt L, Algaba F, Welvaart NY, Beukers W, et al. Molecular markers increase precision of the European association of urology non-muscle-invasive bladder cancer progression risk groups. Clin Cancer Research. 2018;24(7):1586-93.

19. Shindo T, Shimizu T, Nojima M, Niinuma T, Maruyama $\mathrm{R}$, Kitajima $\mathrm{H}$, et al. Evaluation of urinary DNA methylation as a marker for recurrent bladder cancer: A 2-center prospective study. Urology 2018;113:71-8.

20. López J, Angulo J, Martín A, Sánchez-Chapado M, González-Corpas A, Colás B, et al. A DNA hypermethylation profile reveals new potential biomarkers for the evaluation of prognosis in urothelial bladder cancer. APMIS. 2017;125(9):787-96.

21. Husek P, Pacovsky J, Chmelarova M, Podhola M, Brodak M. Methylation status as a predictor of intravesical Bacillus Calmette-Guérin (BCG) immunotherapy response of high grade non-muscle invasive bladder tumor. Biomed Papers 2017;161(2):210-6.

22. Jin F, Thaiparambil J, Donepudi S, Vantaku V, Piyarathna DW, Maity S, et al. Tobacco-specific carcinogens induce hypermethylation, DNA adducts, and DNA damage in bladder cancer. Cancer Prev Res 2017;10(10):588-97.

23. Vantaku V, Amara C, Piyarathna D, Donepudi SR, Ambati CR, Putluri V, et al. DNA methylation patterns in bladder tumors of African American patients point to distinct alterations in xenobiotic metabolism. Carcinogenesis 2019;40(11):1332-40.
24. Huang Y, Shen X, Zou Q, Wang S, Tang S, Zhang G. Biological functions of microRNAs: A review. J Physiol Biochem 2010;67(1):129-39.

25. Rothschild S. MicroRNA therapies in cancer. Mol Cell Ther 2014;2(1):7.

26. Xie Y, Ma X, Chen L. MicroRNAs with prognostic significance in bladder cancer: A systematic review and meta-analysis. Sci Rep. 2017;7(1):41.

27. Chen Z, Zhan Y, Chi J, Guo S, Zhong X, He A, et al. Using microRNAs as novel predictors of urologic cancer survival: An integrated analysis. EBioMedicine 2018;34:94-107.

28. Yang F, Li H, Li T, Zhao Y, Liu Z, Li X. Prognostic value of MicroRNA-15a in human cancers: A meta-analysis and bioinformatics. Biomed Res Int 2019;2019:1-12.

29. Shi H, Yu J, Yu J, Feng Z, Zhang C, Li GY, et al. Diagnostic significance of microRNAs as novel biomarkers for bladder cancer: A meta-analysis of ten articles. World J Surg Oncol 2017;15(1):147.

30. Xiao S, Wang J, Xiao N. MicroRNAs as noninvasive biomarkers in bladder cancer detection: A diagnostic meta-analysis based on qRT-PCR data. Int J Biol Markers 2016;31(3):276-85.

31. Ouyang H, Zhou Y, Zhang L, Shen G. Diagnostic value of MicroRNAs for urologic cancers. Medicine (Baltimore) 2015;94(37):e1272.

32.Zabolotneva A, Zhavoronkov A, Garazha A, Roumiantsev S, Buzdin A. Characteristic patterns of microRNA expression in human bladder cancer. Front Genet 2013;3:310.

33. Lin S, Choe J, Du P, Triboulet R, Gregory R. The $m 6$ a methyltransferase METTL3 promotes translation in human cancer cells. Mol Cell 2016;62(3):335-45.

34. Han J, Wang J, Yang X, Yu H, Zhou R, Lu HC, et al. METTL3 promote tumor proliferation of bladder cancer by accelerating pri-miR221/222 maturation in m6A-dependent manner. Mol Cancer 2019;18(1):110.

35. Lu Q, Liu T, Feng H, Yang R, Zhao X, Chen W, et al. Circular RNA circSLC8A1 acts as a sponge of miR$130 \mathrm{~b} / \mathrm{miR}-494$ in suppressing bladder cancer progression via regulating PTEN. Mol Cancer 2019;18(1):111.

36. Hou G, Xu W, Jin Y, Wu J, Pan Y, Zhou F. MiRNA-217 accelerates the proliferation and migration of bladder cancer via inhibiting KMT2D. Biochem Biophys Res Commun 2019;519(4):747-53.

37. Lin T, Zhou S, Gao H, Li Y, Sun L. MicroRNA-325 is a potential biomarker and tumor regulator in human bladder cancer. Technol Cancer Res Treat 2018;17:536.

38. Tian H, Wang X, Lu J, Tian W, Chen P. MicroRNA-621 inhibits cell proliferation and metastasis in bladder cancer by suppressing Wnt/ $\beta$-catenin signaling. Chem Biol Interact 2019;308:244-51. 
39. Feng Y, Kang Y, He Y, Liu J, Liang B, Yang P, et al. MicroRNA-99a acts as a tumor suppressor and is downregulated in bladder cancer. BMC Urol. 2014;14(1):50.

40. Wu D, Niu X, Tao J, Li P, Lu Q, Xu A, et al. MicroRNA379-5p plays a tumor-suppressive role in human bladder cancer growth and metastasis by directly targeting MDM2. Oncol Rep 2017;37(6):3502-8.

41. Jiang $\mathrm{H}, \mathrm{Bu} \mathrm{Q}$, Zeng M, Xia D, Wu A. MicroRNA-93 promotes bladder cancer proliferation and invasion by targeting PEDF. Urol Oncol Sem Original Investig. 2019;37(2):150-7.

42. Witjes J, Morote J, Cornel E, Gakis G, van Valenberg FJ, Lozano F, et al. Performance of the bladder EpiCheck $^{\mathrm{Tm}}$ methylation test for patients under surveillance for non-muscle-invasive bladder cancer: Results of a multicenter, prospective, blinded clinical trial. Eur Urol Oncol 2018;1(4):307-13. 